\title{
Ultrasound Guided Pneumatic Reduction of Intussusception: A Clinical Experience from Baghdad
}

\author{
Ali Egab Joda ${ }^{1,2, ~ *, ~ W a a d ~ M u h a m m a d ~ S a l i h ², ~ N a w z a t ~ H u s s e i n ~ S h a k a r l y ² ~}$ \\ ${ }^{1}$ Department of Surgery, Mustansiriyah Medical College, Baghdad, Iraq \\ ${ }^{2}$ Department of Pediatric Surgery, Central Child Teaching Hospital, Baghdad, Iraq
}

Email address:

ali.egab.joda@gmail.com (A. E. Joda)

*Corresponding author

\section{To cite this article:}

Ali Egab Joda, Waad Muhammad Salih, Nawzat Hussein Shakarly. Ultrasound Guided Pneumatic Reduction of Intussusception: A Clinical Experience from Baghdad. American Journal of Pediatrics. Vol. 3, No. 6, 2017, pp. 76-82. doi: 10.11648/j.ajp.20170306.13

Received: September 10, 2017; Accepted: September 28, 2017; Published: November 10, 2017

\begin{abstract}
Intussusception is an important cause of small bowel obstruction in children. Non-operative reduction (NOR) is the preferable method of treatment to start with as long as there are no contraindications to its use and where facilities are available. Pneumatic reduction has become a popular therapeutic method for intussusception instead of surgery in many centers. The current study aim to evaluate the success rate of ultrasound guided pneumatic reduction of intussusception and verifying the factors affecting its efficacy as initial experience in Baghdad. A prospective study of 56 eligible patients with confirmed intussusception managed over the period of eighteen months from March 2016 to September 2017 in Baghdad. The procedure was performed under ultrasound guidance after adequate resuscitation. Those patients with unsuccessful first attempt of pneumatic reduction were subjected to second and third attempts before surgical intervention performed. a total 39 male and 17 female were found suitable for pneumatic reduction under ultrasound guide. Successful reduction by air insufflation was achieved in 44 patients $(78.5 \%)$, whereas the procedure was failed in the remaining 12 patients $(21.4 \%)$. There were only two out of 44 patients $(4.5 \%)$ with successful reduction developed early recurrence of intussusception. One patient (2.27\%) developed intestinal perforation. Pneumatic reduction of intussusception under ultrasound guidance is a quick, safe, simple, with a high success rate, radiation-sparing effect. Success rate is highly affected by the duration of symptoms and location of mass. The procedure is not devoid of recurrence risk and possibility of developing pneumoperitoneum.
\end{abstract}

Keywords: Intussusception, Pneumatic Reduction, Ultrasonography

\section{Introduction}

Intussusception is the most frequent cause of bowel obstruction in infants and toddlers and probably the second most common cause of acute abdominal pain after constipation in these age groups [1,2]. The treatment of intussusception is an emergency, by either non-operative or operative method. Delay in treatment will lead to ischemia and necrosis of the intestine, bowel perforation, peritonitis, shock, and possibly death [3].

Non-operative reduction (NOR) is the preferable method of treatment to start with as long as there are no contraindications to its use and where facilities are available [3]. The next logical step after failure of non-operative approach is surgery. Indeed, the choice between the two methods (operative and non-operative) depends on the general condition of the patient, the duration of illness and the availability of skilled radiological service [4]. The currently used techniques for non-operative reduction of intussusception include pneumatic or hydrostatic pressure enemas under fluoroscopic or sonographic guidance [1] with the advantages of decreased morbidity, cost, and length of hospitalization [2]. In present study, the aim is to evaluate the success rate of ultrasound guided pneumatic reduction of intussusception and verifying the factors affecting its efficacy as initial experience in Baghdad.

\section{Patients and Method}

A prospective study included 56 infants and children with idiopathic, uncomplicated intussusception (as inclusion criteria) 
treated by non-operative reduction (NOR) over the period of eighteen months from March 2016 to September 2017 in Baghdad. While patients with ages more than 2 years, delayed presentation ( $>72$ hours), cases of recurrent intussusception, and features of peritonitis or shock were excluded from present study ( $\mathrm{n}=18$ patients). A special data form had been used including variables of age, sex, residence, history of admission to other hospital, clinical presentation, coexisting illness, investigations, type of management in addition to postoperative notes and complications. All patients with suspicion of intussusception were admitted to the surgical ward, clinical evaluation and assessment of the general condition were made by a full history and physical examination, resuscitation was done by insertion of intravenous line, nasogastric tube and starting fluid therapy and antibiotics. The patients were sent for plain abdominal $\mathrm{x}$ ray, ultrasound of the abdomen, and laboratory investigations (complete blood count, blood urea, serum creatinine, and serum electrolytes).

After confirming the diagnosis by ultrasound examination, the definitive treatment started within few hours when the general condition of patient became stable, a cross- matched blood was prepared for all patients regardless of the method used in the treatment, and written informed consent was taken from each patient's parent or guardian for participation in the study. The study was approved by the local hospital ethical committee. Statistical analysis of the data was done using SPSS-version 18 software (IMB Corporation). Using percentage, mean, and ANOVA (Analysis of variance). A p value less than 0.05 was considered statistical significant.

Air Insufflation was Used for NOR in 56 Patients Through the Modified Air Insufflation Device (Figure 1). Surgery was Performed in Patients with Exclusion Criteria or Those Patients with Failed Attempts of Pneumatic Reduction.

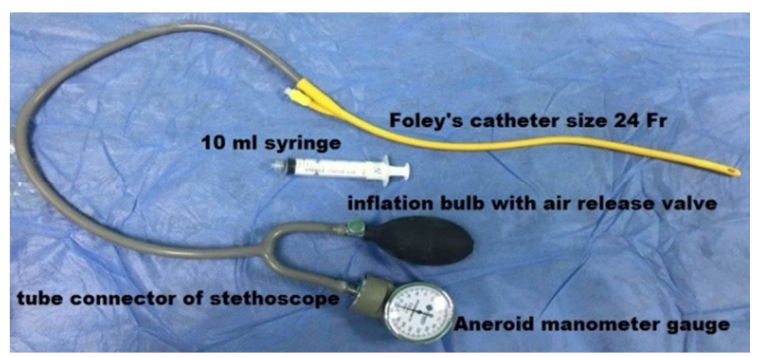

Figure 1. Modified Air Insufflation Device.

The procedure was done under ultrasound guidance without sedation, anesthesia and muscle relaxant by inserting a well lubricated large size Foley's catheter ( $22 \mathrm{Fr}$, or 24Fr) into the rectum and inflating the balloon with $25-30 \mathrm{ml}$ normal saline, then pulled down to be kept as low as possible. With the patient in a supine position, the buttocks were taped together with the help of a nurse. The other end of the catheter was connected to the air insufflation device. The air was introduced into the colon by gradual squeezing the inflation bulb with maintaining pressure between 60 and $120 \mathrm{mmHg}$ (The maximum air pressure for younger infants and in the first attempt of reduction was $100 \mathrm{mmHg}$ while pressure of up to
$120 \mathrm{mmHg}$ was used for older infants and in the second and third attempts of reduction). There is fluctuation in the measured pressure with straining and crying of the child and in between the insufflation. The reduction process is tracked by ultrasound scanning probe throughout the procedure. The increased intracolonic pressure will reduces intussusceptum gradually through the ileo-caecal junction. The procedure is terminated once there is satisfactory clinical and ultrasound features of complete reduction of intussusception. Although successful reduction of intussusception was confirmed by ultrasound, further confirmation by plain abdominal x-ray in some patients was achieved, which showed delineation of the whole colon and terminal ileum with gases. After that, the patient was returned back to the surgical ward and kept there for about 24-48 hours for observation and detection of any complication.

Those patients with failed first trial of reduction, another one to two trials of reduction were performed within the next 2-4 hours. High resistance to air insufflation and Persistence of the mass by ultrasound were the signs of unsuccessful reduction and indications of operative intervention.

Patients with successful reduction were sent for ultrasound examination in the next day to exclude any residual pathology or recurrence and discharged home based on the following criteria:

1. Disappearance of all symptoms and signs of intussusception.

2. Starting oral feeding without vomiting

3. Passing normal bowel motion.

4. Disappearance of the mass by ultrasound.

\section{Results}

Among total 74 patients with intussusception managed during the study period, non-operative reduction by mean of air insufflation was attempted in $56(75.6 \%)$ Patients as initial line of treatment. The majority of patients in present study (49 patients, $87.5 \%$ ) were under the age of one year, the group of 4-9 months age being the commonest age group (39 patients, 69.6\%) (Figure 2). The youngest patient in present study was male aged 3 months while the oldest one was a male aged 22 months. Mean age was 7.77 month. There were 39 males $(69.6 \%)$ and 17 females (30.3\%), the male to female ratio in present study was 2.2:1 (Figure 2).

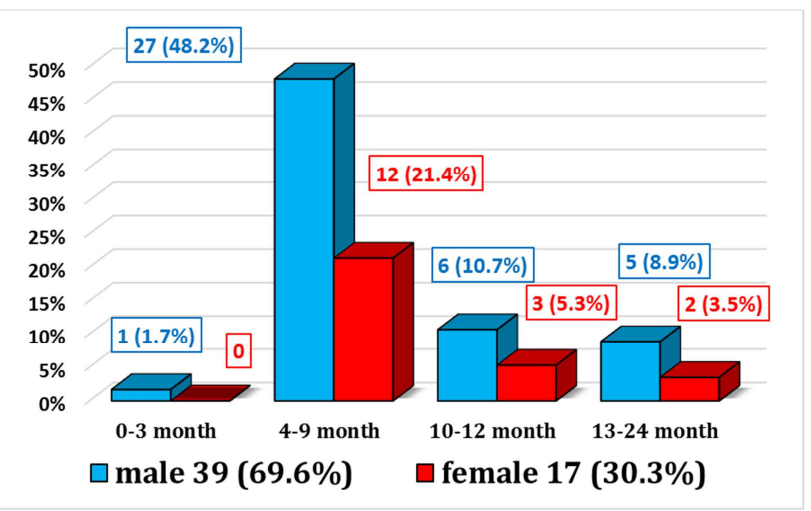

Figure 2. Sex and Age Distribution. 
Figure 3 shows the duration of symptoms prior to presentation to hospital. Only 20 patients (35.7\%) presented early within the first 24 hours of onset of symptoms. Screaming attacks (colicky abdominal pain) and repeated vomiting were the most common presenting symptoms $96 \%$ and $89 \%$ respectively. The family gave history of bleeding per rectum in 46 out of 56 patients $(82 \%)$. On physical examination, abdominal tenderness was obvious in 42 patients $(75 \%)$. palpable abdominal mass was felt in only 30 patients $(53.57 \%)$ while the characteristic red currant jelly stool was noticed following per-rectal examination in 45 patients $(80.3 \%)$. abdominal distention and palpable mass per rectum or prolapsed through anus were found in the advanced cases (more than 48-hour duration of symptoms). The classic clinical triad of abdominal pain, abdominal mass and rectal bleeding occurred only in 31 patients $(55.3 \%)$. (See Table 1)

On clinical and ultrasound examination, the most common site of the mass was in the upper right side of the abdomen in 29 patients $(51.7 \%)$. Central abdominal and epigastric mass was noticed in 15 patients $(26.7 \%)$. While palpable mass in the left side of abdomen was noticed in 12 patients $(21.4 \%)$, five of them having a mass palpable per rectal examination $(8.9 \%)$ and in one case the mass was prolapsed through the anus (Figure 4).

Table 1. Symptoms and Signs.

\begin{tabular}{llll}
\hline \multicolumn{2}{l}{ Clinical presentation } & No. & (\%) \\
\hline \multirow{4}{*}{ Symptoms } & Abdominal pain & 54 & 96.4 \\
& Vomiting & 50 & 89.2 \\
& Bleeding per rectum & 46 & 82.1 \\
& Constipation & 3 & 5.3 \\
& Abdominal tenderness & 42 & 75.0 \\
\multirow{5}{*}{ Signs } & Palpable abdominal Mass & 30 & 53.57 \\
& Red Currant jelly stool & 45 & 80.3 \\
& Abdominal distention & 11 & 19.64 \\
\multirow{5}{*}{$\begin{array}{l}\text { The classical triad of intussusception (colicky pain, } \\
\text { abdominal mass, red Currant jelly stool }\end{array}$} & 5 & 8.9 \\
\hline
\end{tabular}

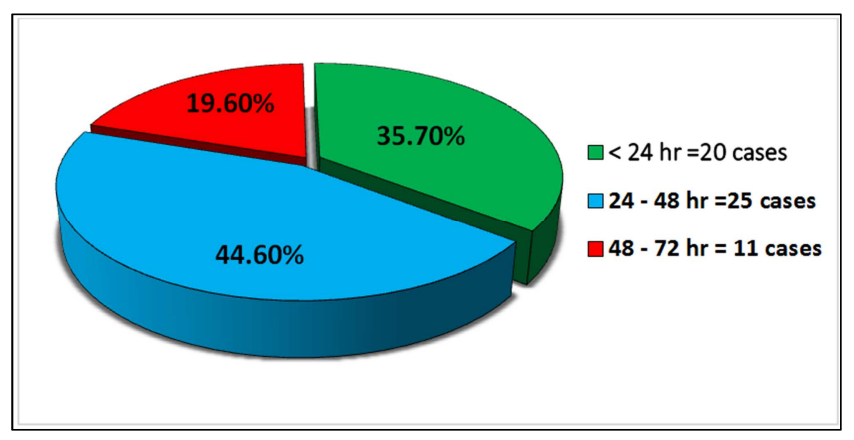

Figure 3. Durations of Symptoms.

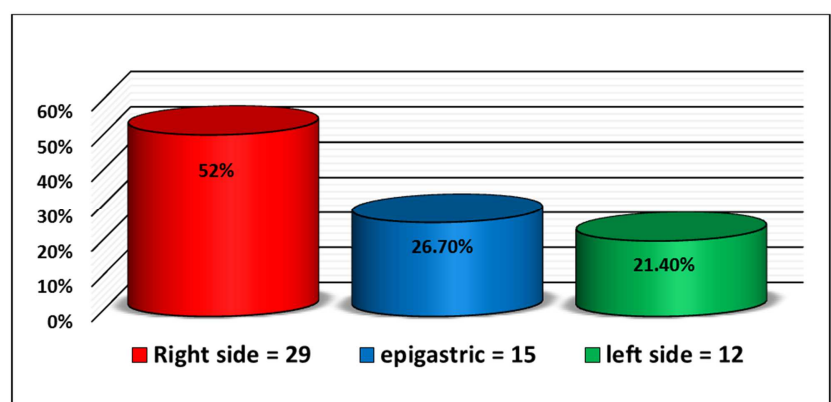

Figure 4. Location of the Mass.

Successful reduction by air insufflation was achieved in 44 patients $(78.5 \%)$, whereas the procedure was failed in the remaining 12 patients $(21.4 \%)$.

On surgical exploration, the bowel at area of intussusception was ischemic in five patients, so underwent resection and end-to-end anastomosis. Difficult manual reduction with some serosal tear was recorded in three patients. Pathological lead points was noticed as a cause of intussusception in the remaining four cases (three cases of Meckel's diverticulum and one case of intestinal lymphoma), and again end with resection and anastomosis.

Regarding relation of duration of symptoms to the successful pneumatic reduction, it was an important predictive factor of successful reduction with highly significant $\mathrm{p}=0.001$. The procedure was highly successful in patients with early presentation (95\%) and least successful in patients with delayed presentation (45.4\%). (Table 2).

Regarding relation of duration of symptoms to the number of attempts of successful reduction, $70 \%$ of those patients who presented early (less than 24 hours) having successful reduction from the first attempt, while $52 \%$ of those patients who presented after 24 hours of symptoms required 2 attempts of pneumatic reduction to achieve complete reduction. Only $18 \%$ of patients with delayed presentation have complete reduction after three attempts. The total rate of successful reduction from the first attempt was recorded in 18 out of 56 patients $(32.1 \%)$, and it was varied considerably according to the duration of symptoms with statistical $\mathrm{P}=$ 0.000 . The total rate of successful reduction from two attempts was recorded in 20 patients (35.7\%) with no statistical variation $(\mathrm{P}=0.111)$ among groups of duration of symptoms. While only six patients (10.7\%) achieved successful reduction with third attempt. $(P=0.013)$. (Table 2$)$

Regarding relation of successful reduction to the location of mass as identified by examination and ultrasound finding at the time of presentation; the procedure achieved high rate of successful reduction in the right sided and epigastric masses $(86.2 \%, 86.6 \%$ respectively) and least rate in the left sided masses or when the mass was palpable per rectum. Again the rate of successful reduction is varied considerably according to mass location $(\mathrm{P}=0.004)$ as shown in (Table 3 ). 
Table 2. Relation of Duration of Symptoms to the Successful Reduction.

\begin{tabular}{|c|c|c|c|c|c|c|}
\hline & & \multicolumn{3}{|c|}{ Duration of symptoms } & \multirow{2}{*}{ Total $(n=56)$} & \multirow{2}{*}{ ANOVA } \\
\hline & & $<24 \mathrm{hr}(\mathrm{n}=20)$ & $24-48 \mathrm{hr}(\mathrm{n}=25)$ & $48-27 \mathrm{hr}(\mathrm{n}=11)$ & & \\
\hline \multirow{4}{*}{ Success rate } & After 1 trial & $14(70 \%)$ & $3(12 \%)$ & $1(9.1 \%)$ & $18(32.1 \%)$ & $0.000^{* *}$ \\
\hline & After 2 trials & $5(25 \%)$ & $13(52 \%)$ & $2(18.1 \%)$ & $20(35.7 \%)$ & 0.111 \\
\hline & After 3 trials & 0 & $4(16 \%)$ & $2(18 \%)$ & $6(10.7 \%)$ & $0.013 *$ \\
\hline & Total (\%) & $19(95 \%)$ & $20(80 \%)$ & $5(45.4 \%)$ & $44(78.5 \%)$ & $0.001 * *$ \\
\hline Failed (\%) & & $1(5 \%)$ & $5(20 \%)$ & $6(54.5 \%)$ & $12(21.4 \%)$ & \\
\hline
\end{tabular}

* Statistically significant when $\mathrm{p}$ value $<0.05$.

** Statistically significant when $\mathrm{p}$ value $<0.01$.

ANOVA $=$ analysis of variance.

Regarding relation of trials of reduction to the location of mass, the author found that a good number of patients $(48.3 \%)$ with right sided mass have successful reduction with single attempt only, while $50 \%$ of those patients with left sided mass or mass that is palpable through rectal exam have failure reduction even after all three attempts of air insufflation. (Table 3).

There were only two out of 44 patients with successful reduction $(4.5 \%)$ developed recurrence of intussusception within less than 48 hours of successful reduction confirmed by ultrasound examination (Figure 5). Repeated air insufflation reduction was done under ultrasound guide and was successful in both cases with single attempt. One patient developed intestinal perforation $(2.27 \%)$ He was 5 months old male infant with more than 48-hour duration of symptoms. The perforation occurred during the first attempt of reduction at pressure of $90 \mathrm{mmHg}$ when the author noticed sudden drop in the pressure and clinical deterioration. It was promptly recognized by the radiologist and the patient proceeded immediately to theatre where surgery done for him and a small perforation in an ischemic area of ascending colon with no peritoneal soiling was discovered. Right hemicolectomy and ileo-colic anastomosis was done and the patient then got full recovery. No mortality rate encountered during the study period.

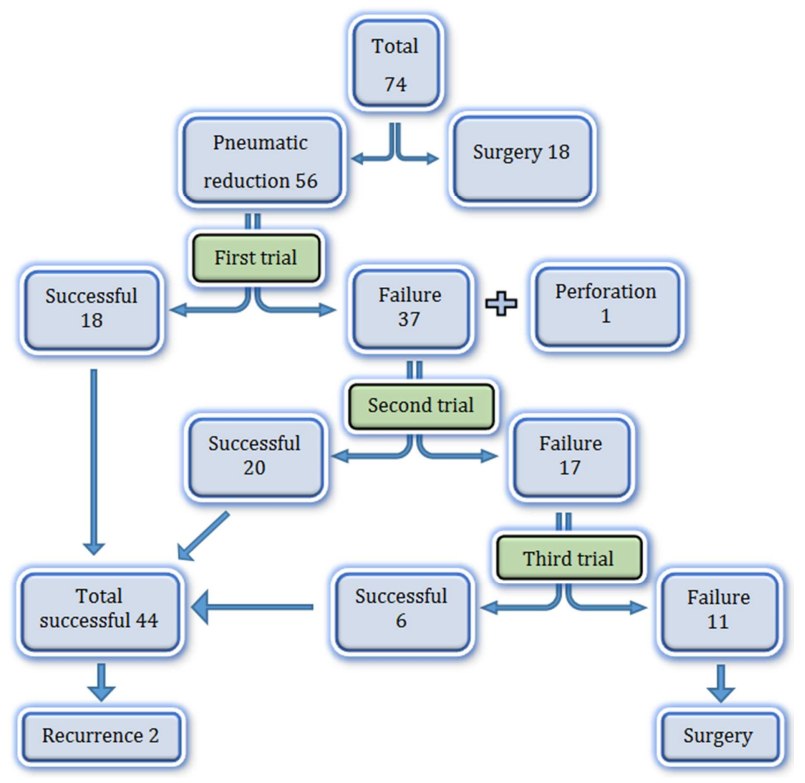

Figure 5. Diagram of Outcomes of Pneumatic Reduction.

Table 3. Relation of Location of Mass to the Successful Reduction.

\begin{tabular}{|c|c|c|c|c|c|c|}
\hline & & \multicolumn{3}{|c|}{ Location of the mass } & \multirow{2}{*}{ Total $(n=56)$} & \multirow[b]{2}{*}{ ANOVA } \\
\hline & & Right side $(n=29)$ & Epigastric $(n=15)$ & Left side $(n=12)$ & & \\
\hline \multirow{4}{*}{ Success rate } & After 1 trial & $14(48.3 \%)$ & $4(26.6 \%)$ & 0 & $18(32.1 \%)$ & $0.002 * *$ \\
\hline & After 2 trials & $9(31.1 \%)$ & $6(40 \%)$ & $5(41.6 \%)$ & $20(35.7 \%)$ & 0.479 \\
\hline & After 3 trials & $2(6.7 \%)$ & $3(20 \%)$ & $1(8.3 \%)$ & $6(10.7 \%)$ & 0.664 \\
\hline & Total (\%) & $25(86.2 \%)$ & $13(86.6 \%)$ & $6(50 \%)$ & $44(78.5 \%)$ & $0.004 * *$ \\
\hline Failed (\%) & & $4(13.7 \%)$ & $2(13.3 \%)$ & $6(50 \%)$ & $12(21.4 \%)$ & \\
\hline
\end{tabular}

** Statistically significant when $\mathrm{p}$ value $<0.01$.

ANOVA $=$ analysis of variance.

\section{Discussion}

Non-operative reduction (NOR) of intussusception is the first-line of treatment if there are no contraindications to its use, it include pneumatic or hydrostatic reduction under fluoroscopic or ultrasound guidance [1]. In contrast to operative reduction, NOR have decreased morbidity, cost, length of hospitalization, and devoid of surgical complications [2]. Pneumatic reduction was first described in 1897 [5] and then gained popularity in the late 1980s when ultrasound guidance has been added to the armamentarium. Since then, many institutions have adopted this procedure because it is quick, safe and decreases the exposure time to radiation [4].

In present study, the author found that the majority of patients $(69.6 \%)$ were in the age group of 4-9 months and this is consistent with what mentioned in the textbooks and literatures $[1,2]$. The male to female ratio was $2.2: 1$, which is similar to the ratio reported by literatures $[6,7]$. Most of the patients were well-nourished and healthy supporting Hirschsprung's classic statement: "I never saw a 
malnourished child with intussusception" [1].

Only 20 of the patients (35.7\%) presented early within the first 24 hours of onset of symptoms while study of Dahab et al. [8] in Cairo and Alhasani [9] in Basra reported that the majority of their patients $(60 \%, 54.3 \%$ respectively) presented early within the first 24 hours of onset of their symptoms. The large number of patients in present study with more than 24 hours duration of symptoms may be attributed to delayed diagnosis in some patients, misdiagnosis (as gastroenteritis, otitis media..... etc.) or due to late referral by the local general practitioner. All of the presenting features in present study were comparable with corresponding features of other studies (as shown in Table 4).

Table 4. Comparison with Other Studies Regarding Symptoms and Signs.

\begin{tabular}{|c|c|c|c|c|c|c|c|}
\hline \multirow[b]{2}{*}{ Symptoms and signs } & \multicolumn{7}{|l|}{ Study } \\
\hline & Dahab et al.[8] & Saleh et al $[10]$ & $\begin{array}{l}\text { Arsalan et } \\
\text { al:[11] }\end{array}$ & $\begin{array}{l}\text { Shahwani } \\
{[12]}\end{array}$ & Zain AZ [13] & $\begin{array}{l}\text { Gata and } \\
\text { Ejrish }[14]\end{array}$ & present study \\
\hline Abdominal pain (\%) & 98 & 85 & 94.6 & 71 & 90 & 100 & 96.4 \\
\hline Vomiting (\%) & 94.5 & 70 & 82.6 & 54 & 87.5 & 78 & 92.2 \\
\hline Bleeding per rectum (\%) & 91 & 80 & 81.3 & 58 & 66.2 & 90 & 82.1 \\
\hline Palpable abdominal Mass (\%) & 11 & 75 & 56.6 & 73 & 60 & 66 & 53.5 \\
\hline Red Currant jelly stool (\%) & NR & NR & NR & 64 & NR & NR & 80.3 \\
\hline Abdominal distention (\%) & 73 & NR & NR & 15 & 5 & NR & 19.6 \\
\hline Palpable mass per rectum (\%) & 7 & 10 & NR & 4 & NR & NR & 8.9 \\
\hline Prolapsing bowel from anus (\%) & NR & NR & NR & 0.5 & 3.75 & 8 & 1.7 \\
\hline
\end{tabular}

$\mathrm{NR}=$ not recorded

Here anesthesia or sedation was not used during pneumatic reduction because it could mask the signs of shock during the procedure [15]. Muscle relaxant was also not used because smooth muscle tension during the reduction procedure protects against bowel perforation. Furthermore the increased intra-abdominal pressure caused by crying and straining help in rapid reduction $[16,17,18]$.

The small bowel which is invaginated into the caecum will be pushed back to its place by air pressure and relieving the obstruction. The ultrasound criteria for successful reduction after deflation of air included disappearance of the intussusceptum, single concentric ring representing the swollen terminal ileum instead of the multiple concentric rings of intussusception, a severely swollen terminal ileum, and the abrupt transition of bowel wall thickness between the swollen terminal ileum and the proximal normal ileum on longitudinal axis scanning [15].

Successful reduction by air insufflation was achieved in 44 patients $(78.5 \%)$. Recent studies in nearby countries reported rates of successful reduction range from $74 \%$ to $94.6 \%$ as shown in (Table 5).

Table 5. Comparison of Success Rate with Other Studies.

\begin{tabular}{|c|c|c|c|c|}
\hline Study & Year & Cases & Country & Success (\%) \\
\hline A. lshahwany [12] & 2001 & 120 & Iraq & 83 \\
\hline Saleh et al. [10] & 2005 & 20 & Egypt & 78 \\
\hline S. Kritsaneepaiboon, et al. [19] & 2011 & 73 & Thailand & 74 \\
\hline Korkmaz et al.[6] & 2012 & 18 & Turkey & 83.3 \\
\hline Dahab et al.[8] & 2012 & 50 & Egypt & 78 \\
\hline Arsalan et al.[11] & 2014 & 150 & Turkey & 94.6 \\
\hline Hosni Morsi Ahmed [20] & 2015 & 132 & Egypt & 81.8 \\
\hline Al-Meflh et al. [7] & 2016 & 45 & Jordan & 88.9 \\
\hline Present study & 2016 & 56 & Iraq & 78.5 \\
\hline
\end{tabular}

It have been found that as the duration of symptoms increases, the rate of successful reduction (especially from first attempt) will decrease. The success rate of pneumatic reduction was $95 \%$ when the patients presented early within the first 24 hours of symptoms and decreased to $45 \%$ when the patients presented lately after 48 hours of stating the symptoms. This have a statistical significant $\mathrm{P}$ value $(0.001)$. The same thing was reported by other literatures $[8,19]$. The long duration of symptoms will give time to development of edema and vascular changes that make the intussusception so tight and decreases the likelihood of pneumatic reduction. Here in the duration of symptoms also correlate with the number of trials needed to achieve successful reduction, $70 \%$ of those patients who presented early achieved successful reduction with first attempt compared to $9 \%$ of those with delayed presentation, (statistical significant $\mathrm{p}$ value 0.000 ). (Table 2)

The location of mass can predict the chance of successful reduction and the number of trial needed to achieve it. The more distally located mass of intussusception have less rate of successful reduction with statistical significant $P=0.004$. This is similar to that reported by S. Kritsaneepaiboon, et al. [19].

In general, about one third of all patients under the study have successful first attempt of reduction, which is much lower than that reported by the others like Salih et al. [10] (85\%), Arsalan et al. [11] (91\%), Kritsaneepaiboon, et al. [19] (76\%), Hosni et al. [20] (58.3\%), and Omar Bin Hassan 
et al. [21] 79\%. This low success rate of first attempt reduction in present study may be due to the low pressure applied initially and the less experience with this modality of treatment in our department. In the second attempt, the author increased the pressure to $120 \mathrm{mmHg}$ so there was a higher success rate of $35.7 \%$ which is higher than that reported by the Dahab et al. (12\%) [8]. The low pressure used initially in the first attempt can explain this. In the third attempts, the success rate in present study was $10.7 \%$, which was similar to that reported by the others. For unsuccessful reduction with first attempt, several studies have shown improved reduction rates using a second attempt after waiting between 30 minutes to 24 hours after the initial attempt [22]. In some instances, this is done in the operating room prior to laparoscopy or in conjunction with laparoscopic reduction [23].

Several risk factors have been found in literature to decrease the success rate of pneumatic reduction [1] such as younger ages (less than three months), longer duration of symptoms (more than two days) and the type of intussusception; Enema reduction has a higher failure rate in patients with ileoileocolic intussusception [1]. Ultrasonographic findings of trapped fluid within the intussusception and the intussusceptum reaching the rectum are other conditions associated with less successful enema reduction [24].

Here in present study, immediate recurrence of intussusception was recorded in (4.5\%). The recurrence rate following initial NOR is reported to be as high as $10 \%$ to $15 \%$ of patients. But, it is much lower following surgical reduction (1\% to $3 \%$ ) [25-28]. Indeed, adhesions created by the operative manual reduction, and ileocolic resection are factors responsible for this lower recurrence rate after surgical reduction [29]. Recurrence following NOR may be due to incomplete reduction (but that is less likely under fluoroscopy or ultrasound guided reduction) or due to the presence of a pathological leading point. Niramis et al. reported about a 3 -fold increase in the incidence of lead points in children with recurrent intussusception. There was no reported cases of pathological lead points in the two patients with recurrence and they were treated successfully with a further pneumatic reduction. Current concepts suggest that the initial management of recurrent intussusceptions should be non-operative treatment, even if they recur after previous operative reduction [29, 30].

Bowel perforation occurred only in one patient who presented lately after 2 days of onset of symptoms. Although repeated or delayed second attempt of reduction is a risk factor for bowel perforation [31], the late presentation appear to be more risky according to our data. Dahab et al. [8] reported one case of perforation on the third attempt of reduction after 10 hours from the first attempt, while in present study all of the second and third trials were performed within the next 2-4 hours from the initial attempt as recommended by others [31]. No mortality has been encountered during the procedure.

The ultrasound guidance has added the advantages of avoiding radiation exposure, providing more information about monitoring of reduction process, visualizing all components of intussusception including the post-reduction edematous ileocecal region. However, ultrasonography has some limitations; it is operator-dependent so that an expert radiologist or adequate training is required. Furthermore, it has low specificity in detecting pneumoperitoneum [32] and need ultrasound machines with high-quality images to detect intra-peritoneal free air [33].

\section{Conclusion}

In summary, ultrasound guided pneumatic reduction of intussusception is feasible in all children less than 2 years unless contraindicated. The procedure can be done without sedation or general anesthesia safely and has high success rate, radiation-sparing effect, minimal risk of bowel perforation with less peritoneal contamination, moreover accurate pressure measurement is possible. The success rate is highly affected by the duration of symptoms and location of mass, so that early diagnosis and referral to pediatric surgical centers will improve the results. Second and third attempts of reduction are encouraged after unsuccessful initial attempt. The procedure is not devoid of recurrence risk and possibility of developing pneumoperitoneum.

\section{Conflict of Interest}

None. I wish to confirm that there are no known conflicts of interest associated with this publication and there has been no significant financial support for this work that could have influenced its outcome.

\section{References}

[1] Columbani PM, Scholz S. Intussusception. In: Coran AG, Adzick NS, Thomas Rummel TM et al. pediatric surgery. Volume 2. Seventh edition. Philadelphia; 2012. P: 1093-1110.

[2] Maki CA, Fallat ME. Intussusception. In: George W. Holcomb, J. Patrick Murphy, Daniel J. Ostlie. Ashcraft pediatric surgery. 6th ed. Elsevier, Saunders; 2014. P: 531-538.

[3] Hesse AAJ, Abantanga FA, Lakhoo K. Intussusceptions. In: Ameh EA, Bickler SW, Lakhoo K, et al. Pediatric surgery: comprehensive text for Africa; Vol 2; 2011. P: 404-411.

[4] Guo JZ, Ma XY, Zhou QH. Results of air pressure enema reduction of intussusception: 6,396 cases in 13 years. J Pediatr Surg 1986; 21: 1201-3.

[5] Holt, LE. The diseases of infancy and childhood. In: D. Appleton, New York; 1899: 816-818.

[6] Korkmaz M, Yazgan H, Budan K et al. Pneumatic reduction in the treatment of childhood intussusception cases. Gaziantep Medical Journal. 2012; 18 (2): 56-60.

[7] Al-Meflh W, Abu Quraa A, Khaswneh G et al. Pneumatic Reduction of Pediatric Intussusception: Experience at Queen Rania Al-Abdullah Hospital for Children. Journal of the Royal Medical Services. 2016; 23 (3): 13-19 /DOI: 13-18/ DOI: $10.12816 / 0029068$. 
[8] Dahab MM, AbouZeid AA, Mohammad SA. The second trial pneumatic reduction for idiopathic intussusception: therapeutic effect and hazards. Annals of Pediatric Surgery. 2012; 8 (3): 77-9.

[9] Alhasni AA. Assessment of intraoperative manual reduction of intussusception in children. Bas J Surg, December 22, 2016. P. 69-76.

[10] Saleh G, El-saket MD, Eassa O et al. Pneumatic reduction versus surgical reduction in cases of neonatal inutssusception. El-minia med., Bull., vol. 16, no. 1, Jan 2005, P: 39-48.

[11] Arslan S, Turan C, Doganay S et al. The effectiveness of pneumoreduction for intussusception. Ann Ital Chir. $2014 \mathrm{Jul}$; 28: 85 .

[12] Al-Shahwani AR; The use of Air insufflation In managing Intussusception; J. Fac. Med., Baghdad 2001, Vol. 43, No. 2, P. 173-178.

[13] Zain AZ. Management of intussusception in children. Iraqi J. Comm. Med., no. 1, 2012. P. 40-43.

[14] Gata HK and Ejrish AA. The role of ultrasound in diagnosis of intussusception in children in Baghdad 2012-2013. MJB, vol. 13, no. 1, 2016, P. 85-94.

[15] Yoon C. H., Kim H. J., Goo H. W. Intussusception in children: US-guided pneumatic reduction - initial experience. Radiology 2001; 218: 85-88.

[16] Rosenfeld K, McHugh K. Survey of intussusception reduction in England, Scotland and Wales: how and why we could do better. Clin Radiol 1999; 54: 452-8.

[17] Zheng JY, Frush DP, Guo JZ. Review of pneumatic reduction of intussusception: evolution not revolution. J Pediatr Surg 1994; 29: 93-97.

[18] Zulfiqar MA, Noryati M, Hamzaini AH et al. Pneumatic reduction of intussusception using equipment readily available in the hospital. The Medical journal of Malaysia. 2006 Jun; 61(2): 199-203.

[19] Kritsaneepaiboon S, Sankhathat S, Kanngurn S. Pneumatic reduction of intussusception: factors affecting outcome in Thailand. Asian Biomedicine Vol. 5, no. 2, April 2011; P: 235241.

[20] Ahmed HM, Ahmed O, Ahmed RK. Adding a custom made pressure release valve during air enema for intussusception: A new technique. African Journal of Paediatric Surgery: AJPS. 2015; 12 (4): 232-235. doi: 10.4103/0189-6725.172550.
[21] Hasan OB, Farres SN, Ibrahim M. Ultrasound guided pneumatic reduction of intussusception in children - A case series. Int J Recent Sci Res 2015; 6: 4204-7.

[22] Daneman A, Navarro O. Intussusception. Part 1: A review of diagnostic approaches. Pediatr Radiol. 2003; 33: 79-85.

[23] Kao C, Tseng SH, Chen Y. Laparoscopic reduction of intussusception in children by a single surgeon in comparison with open surgery. Minim Invasive Ther Allied Technol 2011; 20: 141-5.

[24] Yamout SZ, Caty MG, Glick PhL. Complications of Surgery of the Stomach, Duodenum, and Small Intestine. In: Complication in pediatric surgery. Caty MG, Glick $\mathrm{PhL}$, Levitt MA, et al. Informa Healthcare, New York; 2009. P: 257-285.

[25] Champoux AN, Del Beccaro MA, Nazar-Stewart V. Recurrent intussusception risks and features. Arch Pediatr Adolesc Med 1994; 148: 474-8.

[26] Stringer DA, Ein SH. Pneumatic reduction: advantages, risks and indications. Pediatr Radiol 1990; 20: 475-7.

[27] Renwick AA, Beasley SW, Phelan E. Intussusception: recurrence followings (oxygen) enema reduction. Pediatr Surg Int 1992; 7: 361-3.

[28] Daneman A, Alton DJ, Lobo E et al. Patterns of recurrence of intussusception in children: a 17-year review. Pediatric radiology. 1998; 28(12): 913-9.

[29] Niramis R, Watanatittan S, Kruatrachue A, et al.. Management of recurrent intussusception: nonoperative or operative reduction? J Pediatr Surg 2010; 45(11): 2175-2180.

[30] Kaiser AD, Applegate KE, Ladd HP. Current success in the treatment of intussusception in children. Surgery 2007; 142: 469-77.

[31] Sandler AD, Ein SH, Connolly B et al. Unsuccessful airenema reduction of intussusception: is a second attempt worthwhile? Pediatr Surg Int (1999) 15: 214. https://doi.org/10.1007/s003830050558.

[32] Chen SC, Wang HP, Chen WJ et al. Selective Use of Ultrasonography for the Detection of Pneumoperitoneum. Academic Emergency Medicine 2002, 9: 643-645. doi: 10.1197/aemj.9.6.643.

[33] Hefny AF, Abu-Zidan FM: Sonographic diagnosis of intraperitoneal free air. J Emerg Trauma Shock 2011, 4(4): 511-3. 